\title{
Review of: "Continuing inequalities in COVID-19 mortality in England and Wales, and the changing importance of regional, over local, deprivation"
}

\author{
Karyn Morrissey ${ }^{1}$ \\ 1 Technical University of Denmark
}

Potential competing interests: The author(s) declared that no potential competing interests exist.

Please see specific comments below:

1. The authors write: 'For our purposes this

represents an improvement, as we are interested in clustering of deaths due to COVID-19, not in clustering of deaths involving COVID-19. High numbers of deaths involving COVID-19 may indicate high COVID-19 prevalence amongst those who died, rather than that such COVID-19 prevalence caused death.'

What do you mean? Please explain exactly why this is an improvement.

2. The authors write: 'We treat non-COVID mortality as a comparator group to explore whether any spatial structure detected in our results is unique to COVID-19 mortality or capturing spatial variation in all-cause mortality. Whilst this cannot be considered a true negative control [14], as it is not independent of our outcome of interest due to competing risks, it can help us contextualise the spatial structure of COVID mortality.'

Please remove the reference to a control. It is so not, so why even mention that it isn't!

3. Why did you chose the three different geographical scales? I really see no narrative on why, except data is now available. This in a way relates to my overall query of the paper, which is why do this at all? What is added by this information?

4. The authors write: 'We are also interested in how the relationship between deprivation and COVID-19 mortality changed over the study period. Moreover, we are interested in whether it is more important whether a neighbourhood is deprived compared to local neighbourhoods, or whether it is the fact that the neighbourhood sits within a deprived region that drives observed associations'.

Why is this important to know? 
